\section{CRYSTAL STRUCTURE OF THE C/EBPa bZIP DOMAIN BOUND TO DNA}

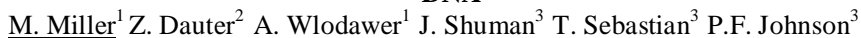
${ }^{1}$ Macromolecular Structure Laboratory, National Cancer Institute P.O. Box B, 7th Str. Blgd. 539 FREDERICK MD 21702-1201 USA ${ }^{2}$ National Synchrotron Light Source, BNL ${ }^{3}$ Regulation of Cell Growth Laboratory, NCI at Frederick

(bZIP) DNA-binding proteins play key roles in regulating gene transcription. Their DNA binding domains consist of the basic region juxtaposed to a heptad repeats of leucines. bZIP proteins from the same family recognize a unique palindromic DNA motif. bZIP peptide binds to DNA as a dimer of $\alpha$ helices. Dimerization is mediated by the leucine zipper; each basic region contacts a half-site in the major groove of the DNA. We solved the crystal structure of the C/EBP $\alpha$ bZIP peptide bound to a 21-mer DNA duplex. The current R-factor is $22.42 \%$ ( $\mathrm{R}$-free is $28.06 \%$ ) in the 40 to $2.8 \AA$ resolution range. Residues conserved among bZIP proteins - Asn292, Ala295 and Arg300 - make DNA contacts that are analogous to those observed in GCN4, CREB and Fos/Jun. Critical for the specific DNA binding by C/EBP proteins is the conformation of Arg289, which makes electrostatic interactions with Asn292, Asn293, and the adenine at position 3 (A3) in the consensus half-site (GCAAT). Val296 makes van der Waals contact with the methyl group of thymine which forms a base pair with A3. We also show that mutation of Arg289 to Ala completely abolishes the C/EBP $\alpha$ DNA binding, whereas a mutant in which Val296 is changed to Ala binds with comparable affinity to the C/EBP and CREB (halfsite $=$ GTCAT $)$ sites.

\section{Keywords: PROTEIN-DNA COMPLEX BZIP C/EBP}

Acta Cryst. (2002). A58 (Supplement), C275

CRYSTAL STRUCTURE OF CbnR, A LysR-TYPE

\section{TRANSCRIPTIONAL REGULATOR}

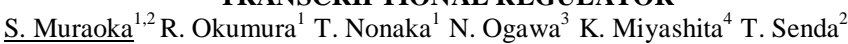
Biological Information Research Center, AIST Aomi 2-41-6, Koto-Ku TOKYO 135-0064 JAPAN

${ }^{1}$ Nagaoka University of Technology ${ }^{2}$ BIRC, National Institute of Advanced Industrial Science and Technology ${ }^{3}$ National Institute for Agro-Environmental Sciences ${ }^{4}$ Agriculture, Forestry, and Fisheries Research Council

LysR-type transcriptional regulators are the most common types of positive regulators in prokaryotes. CbnR, one of the LysR-type transcriptional regulators, regulates the cbnABCD operon in Ralstonia eutropha $\mathrm{NH}$, which encodes the enzymes responsible for the degradation of chlorocatechol. It has been found that CbnR specifically binds to the promoter of the cbnA gene and positively regulates the transcription of cbnABCD in the presence of inducers. In order to elucidate the structure-function relationship of the LysR-type transcriptional regulators, we solved the crystal structure of full-length CbnR. This is the first crystal structure of full-length LysR-type transcriptional regulators. The crystal structure of CbnR was initially solved at $2.7 \AA$ resolution by the MAD method with selenium. The crystallographic refinement is in progress with diffraction data at a $2.0 \AA$ resolution. CbnR is composed of two domains, the DNA-binding and cofactor-binding domains, which are connected by a long $\alpha$-helix with ca. 30 amino acid residues. The DNA-binding domain, which is the $\mathrm{N}$-terminal part of the molecule, consists of three $\alpha$ helices and two antiparallel $\beta$-strands. The cofactor-binding domain has essentially the same folding as that of CysB. It has been proposed that CbnR functions as a dimmer. In the crystal, two possible dimmer forms were found. It remains unclear, however, which possible dimer in the crystal is biologically active. One possible dimer uses the long $\alpha$-helix as a dimer interface, and the other uses the cofactor-binding domain to form the dimer.

\section{Keywords: CRYSTAL STRUCTURE CBNR LYSR FAMILY}

\section{Acta Cryst. (2002). A58 (Supplement), C275}

\section{Acta Cryst. (2002). A58 (Supplement), C275 \\ STRUCTURAL BASIS FOR DNA MISMATCH RECOGNITION BY THE ENZYME MutS}

G. Natrajan M H Lamers H H K Winterwerp A Perrakis T K Sixma The Netherlands Cancer Institute Div of Molecular Carcinogenesis Plesmanlaan 121, AMSTERDAM 1066 CX NETHERLANDS

Introduction: Genomic stability in all organisms depends on their ability to repair DNA mismatches, which come about as a result of polymerase errors. The mismatch repair process is commenced by the enzyme MutS, which recognizes and binds to DNA mismatches and short insertion-deletion loops. Then a number of downstream processes are initiated, leading to the repair of the mismatch.

Aims of the project: MutS binds DNA mismatches and short insertion-deletion loops. We are interested in how this enzyme is able to discriminate between mismatches and Watson-Crick base pairs. To do this, we are determining the crystal structures of the enzyme in complex with oligos containing different mismatches.

Results: Our group has solved the structure of E. coli MutS in complex with a DNA oligomer containing a G:T mismatch. This structure shows a widely conserved phenylalanine, Phe 36, stacking with one of the mispaired bases. The DNA is sharply kinked, changing the way in which the mismatched bases are paired in the absence of the protein. We have now obtained crystals of MutS in complex with A:A, G:G, C:A and G:A mismatches and have solved the structure of MutS in complex with an A:A mismatch. Here too, the kinking of the DNA by the protein changes the base-pairing scheme of the two mispaired adenosines. Thus, it appears that MutS distorts each mismatch and uses the increased conformational flexibility of the mismatch to discriminate between mismatches and Watson-Crick base pairs.

\section{Keywords: DNA MISMATCH REPAIR}

THE CRYSTAL STRUCTURE OF THE SINGLE-STRANDED DNABINDING PROTEIN FROM MYCOBACTERIUM TUBERCULOSIS $\underline{\text { M. Vijayan }}{ }^{1}$ J. Jeyakanthan ${ }^{1}$ J. Venkatesh ${ }^{2}$ N. Acharya ${ }^{2}$ K. Sekar ${ }^{3}$ U. Varshney $^{2}$ K. Saikrishnan ${ }^{1}$

${ }^{1}$ Indian Institute of Science Molecular Biophysics Unit Molecular Biophysics Unit Indian Institute of Science BANGALORE KARNATAKA 560012 INDIA ${ }^{2}$ Department of Microbiology and Cell Biology, Indian Institute of Science, Bangalore 560012 , India ${ }^{3}$ Bioinformatics Centre, Indian Institute of Science, Bangalore 560 012, India

Single-stranded DNA-binding proteins occur widely in viruses, eubacteria, archea and eukaryotes, and are involved in coating single-stranded DNA to protect its chemical and structural integrity. The crystal structures of the proteins from E. coli (EcSSB) and the human mitochondria have been reported. As part of a TB structural genomics project with special emphasis on recombination and repair, the protein from Mycobacterium tuberculosis (MtSSB) has been crystallized in this laboratory in two forms. One of them, trigonal $P 3_{1} 21$, contains the intact protein while the other, orthorhombic $I 222$ or $I 2_{1} 2_{1} 2_{1}$, is made up of truncated proteins resulting from proteolytic cleavage. Attempts at structure solution using molecular replacement did not succeed presumably on account of insufficient sequence and structural similarity with the search models. The structure of the trigonal form has now been solved at $2.6 \AA$ resolution using isomorphous and anomalous data from single site mercury and cadmium derivatives. The tetrameric molecule is located on a crystallographic twofold axis. As expected, the subunits contain the OB fold but their structures differ in detail from those in the other SSBs of known structure. As in EcSSB, with which MtSSB has a somewhat low (28\%) sequence identity, the tetrameric molecule has approximately 222 symmetry, but there are considerable differences in quarternary association and intersubunit interactions.

Keywords: PROTEIN-DNA INTERACTIONS STRUCTURAL GENOMICS OB FOLD 